\title{
Fetal Echocardiography; Introduction and Approaches
}

\section{ART ICLE INF O}

\section{Article Type}

Systematic Review

\section{Authors}

Mosaed P* $M D, P h D$

\section{How to cite this article}

Mosaed P. Fetal Echocardiograp-

hy; Introduction and Approaches.

Sarem Journal of Reproductive

Medicine. 2018;2(1):31-34
* Sarem Fertility \& Infertility Research Center (SAFIR), Sarem Women's Hospital, Tehran, Iran

\section{Correspondence}

Address: Sarem Women's Hospital, Basij Square, Phase 3, Ekbatan Town, Tehran, Iran. Postal Code: 1396956111

Phone: +98 (21) 44670888

Fax: +98 (21) 44670432

pasha_viuna@yahoo.com

\section{Article History}

Received: August 18, 2016

Accepted:December 24,2016

ePublished: February 15, 2018

\section{A B S T R A C T}

Introduction Currently, congenital heart anomalies are the most common anomalies at birth with the incidence rate 8 to 10 per 1000 live births. Fetal heart is small and mobile. The placement of embryos in uterus does not always allow us to take standard images. So, we are faced with an immense variety of images that make it difficult to interpret the abnormal form normal situations (especially in Axis off images). Fetal heart echocardiography is a method to diagnose congenital heart disorders and helps us check fetal heart structure and detect many of the major disorders before birth. The best time for fetal echocardiography is 18 to 24 weeks of gestational age. It can be carried out from the14th week if there is echocardiography device with high sensitivity and accuracy as well as enough experience.

Conclusion The identification and diagnosis of accompanied syndromes, fetal treatments start, the decision to terminate or continue pregnancy, and emotional support of the mother are among the cases that remark the significance of the diagnosis of fetal anomalies.

Keywords Echocardiography; Heart; Fetus; Pregnancy 
غيره شناسايى و تشخيص داده شوند.

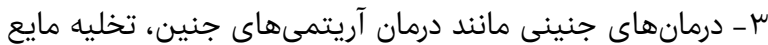
يريكارد و غيره شروع شود.

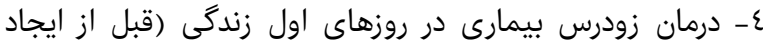

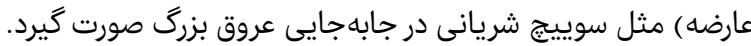

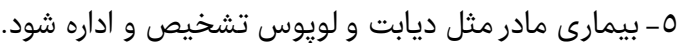

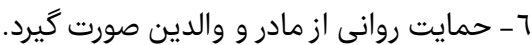

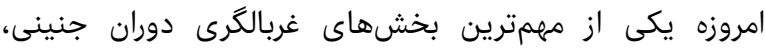

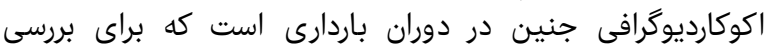

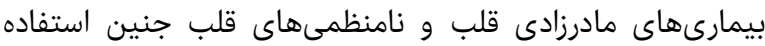

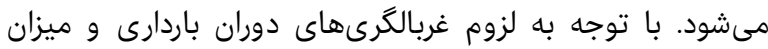

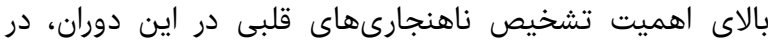

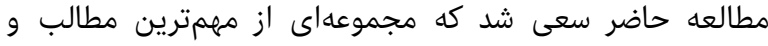

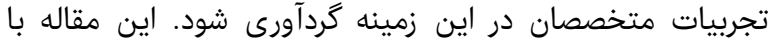

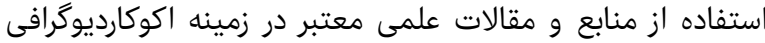

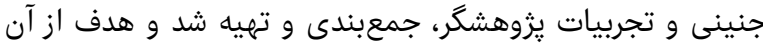

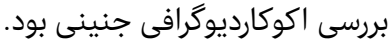

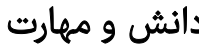

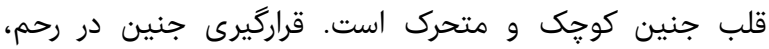

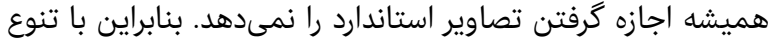

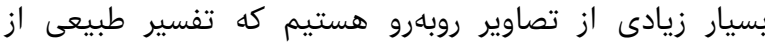
غيرطبيعى را مخصوصاً در تصاوير محور خاموش ارو (Off Axis)، مشكل مىكند.

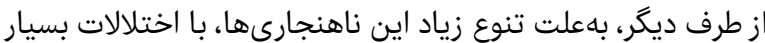

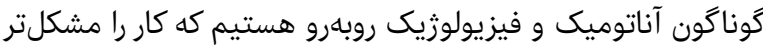
مىكند. در نتيجه انجام و تفسير اكوكارديوگرافى، نيازمند داشتن

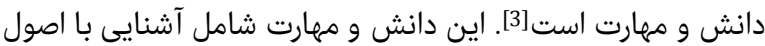

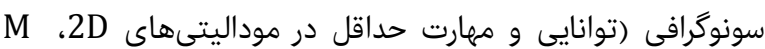
mode فيزيولوزى گردش خون جنين، تمرين و تمركز در داشتن تصور

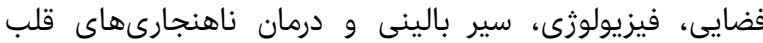

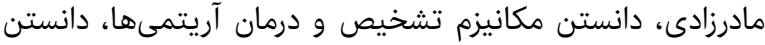

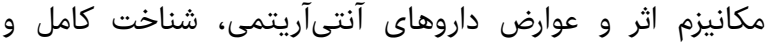

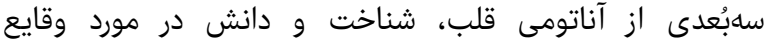

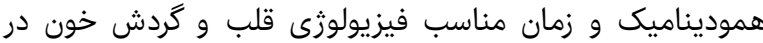

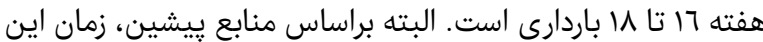

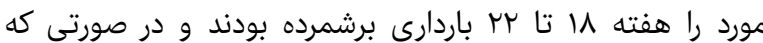

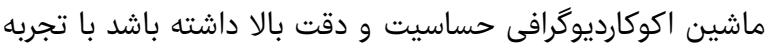

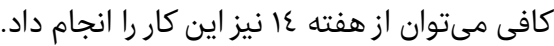

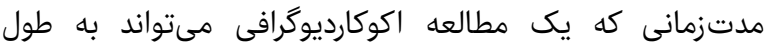

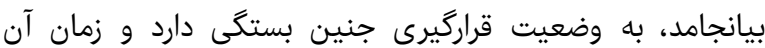

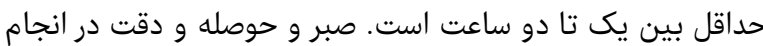

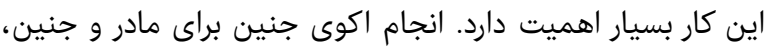

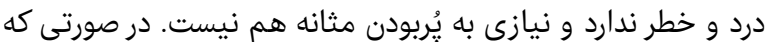

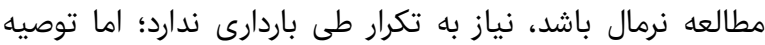

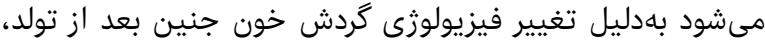

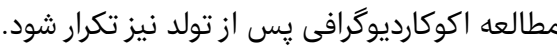

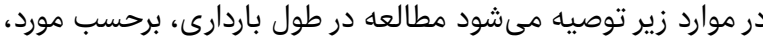

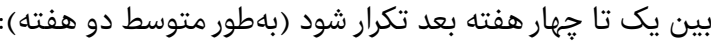

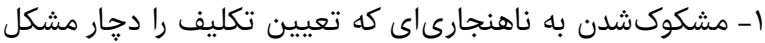

\section{اكوكارديوگرافى جنينى؛ معرفى و خط مشى}

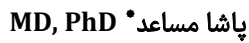

مركز تحقيقات بارورى و نابارورى صارم، بيمارستان فوق تخصصى صارم، تهران، ايران

جكيده مقدمه: در حال حاضر ناهنجارىهاى قلبى مادرزادى شايعترين آنومالىهاى

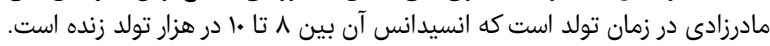

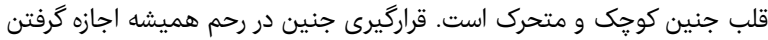

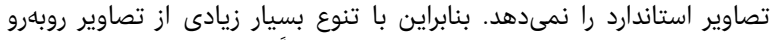

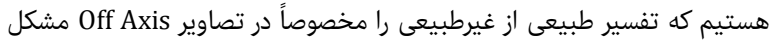

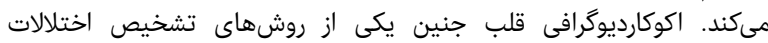

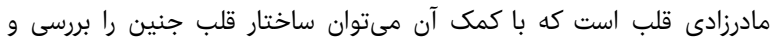

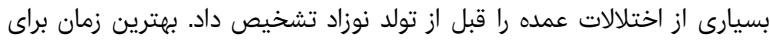

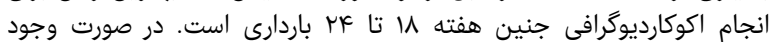

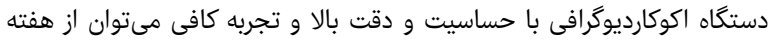

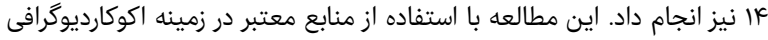

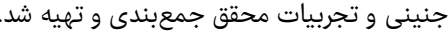

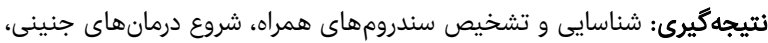

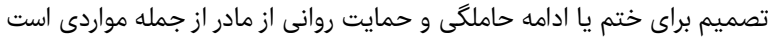

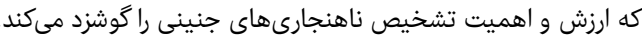
كليدوازهها: اكوكارديوگرافى، قلب، جنين، باردارى اهن

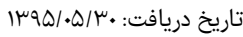

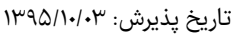
"نويسنده مسئول: pasha_viuna@yahoo.com

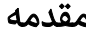

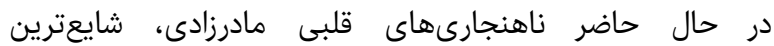

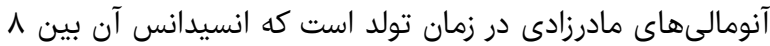

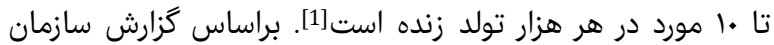

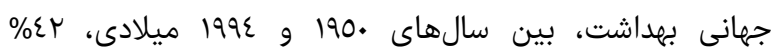

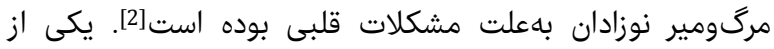

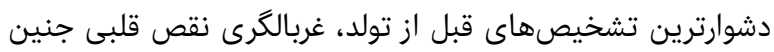

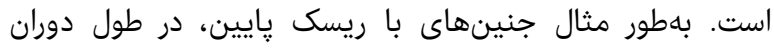

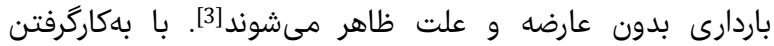

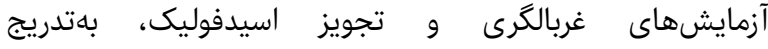

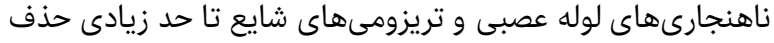

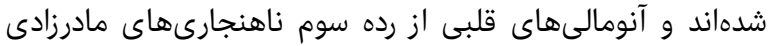

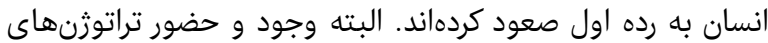

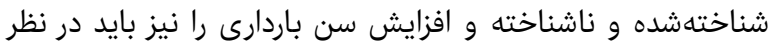

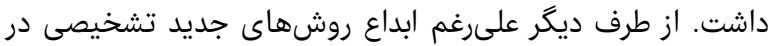

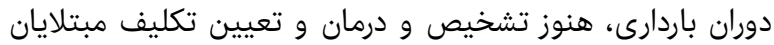

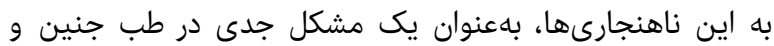

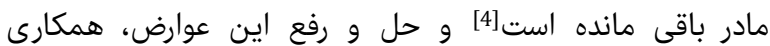

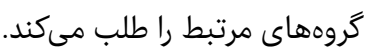

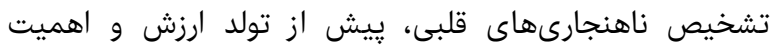

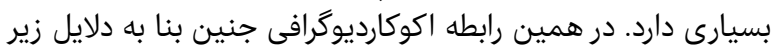

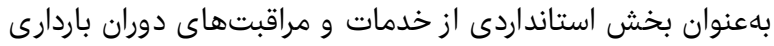

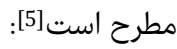

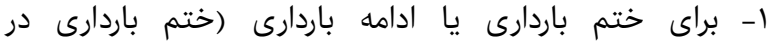

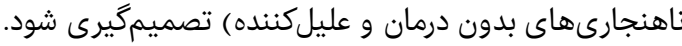

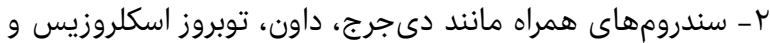


اكوكارديوكرافى جنينى؛ معرفى و خط مشى سر

نديدن نماهاى استاندارد قلبى مثل نماى ع حفره و و سايتوسئر غيرطبيعى بررسى شوند. 9- دوقلويى به بالا، مخصوصاً اگر ترانسفوزيون قلبه بهقل مطرح

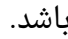

شايعترين ناهنجارىهاى خارج قلبى در جنين كه بايد وضعيت قلب را هم مد نظر داشت به قرارئ زارئ زير است

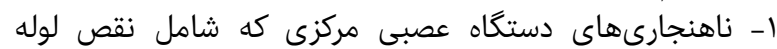

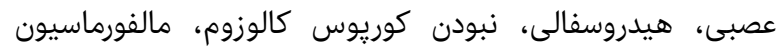

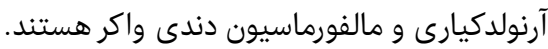

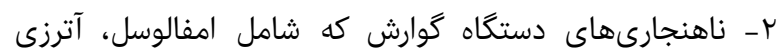

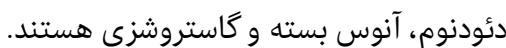

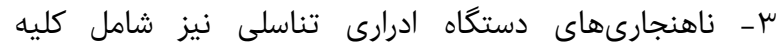
ديسيلاستيك يا فقدان كليه، كليه نعل اسبى و انسداد مجارى إسى نيز هستند. F- ناهنجارىهاى قفسه صدرى كه شامل فيستول مرى به ناى،

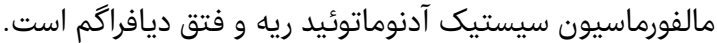

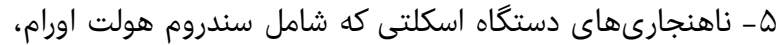

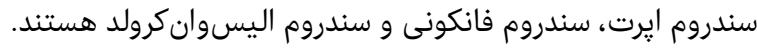

\section{روش مطالعه و دستخاه سونوگرافى}

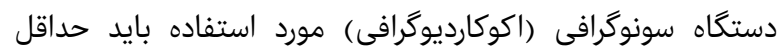

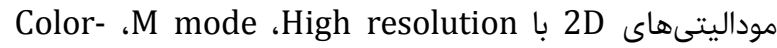
ضو و Pulse-Doppler را داشته باشد. بهعلت بالابودن ضربان قلب جنين (FHR) دستكاه بايد قابليت فركانس تكرار

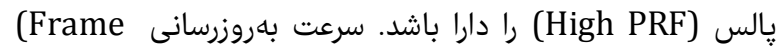

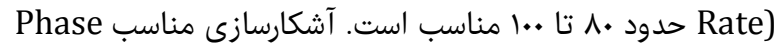
Array Convex (4-12 MHz)

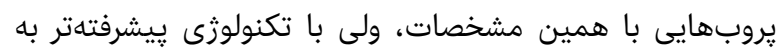
بازار عرضه شدهاند باند. در تكنيك مطالعه، هدف بارة بايد بهدستآدردن همه نماهاى استاندارد

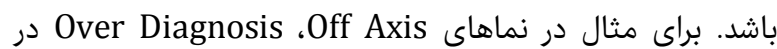

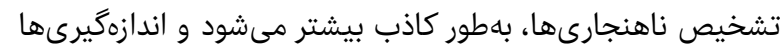

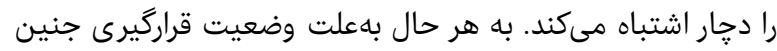

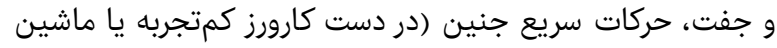

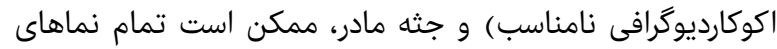

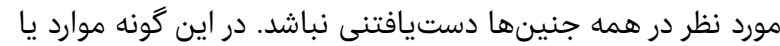

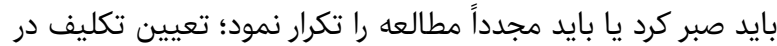

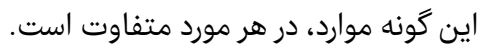

نماهاى مورد مطالعه نماهاى مورد مطالعه، كه براى تشخيص مورد مالعه يا رد ناهنجارىهاى قلبى 1- Upper abdomen sagittal view (V)

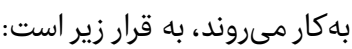

2- 4 Chamber view (V)

3- 5 Chamber view (V)

4- LVOT Long Axis (LX)V

5- RVOT LX V

6- Great Arteries Short Axis (SX)V

7- 3 Vessels V

8- 3 Vessels Tracheal V

9- Bi caval V

10- Ventricular Sx V
ساخته است.

r- نمايش ضعف بيمار كه معمولاً ناشى از ساختمان و جثثه مادر

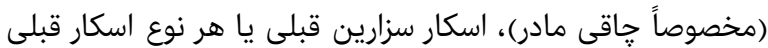

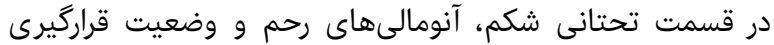
جفت و جنين است.

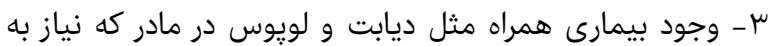
تكرار مطالعه است.

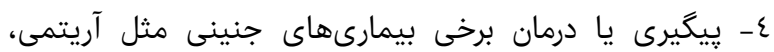

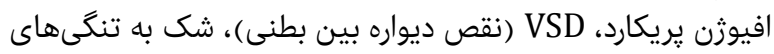
دريجهاى و غيره.

\section{انديكاسيونها} همان طور كه اشاره شد، اكوكارديوگرافى، از ملزومات إنات استاندارد

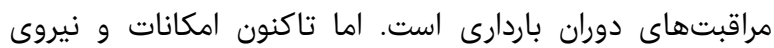

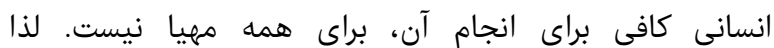

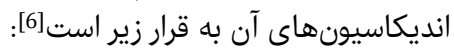

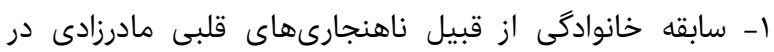

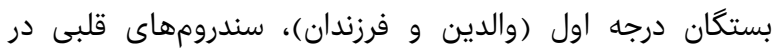

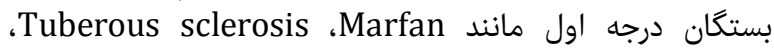
اختلات اختلالات و آنومالىهاى كروموزومى و بيمارىهاى توارثى در

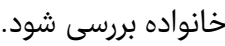

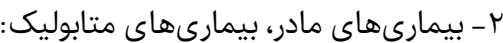

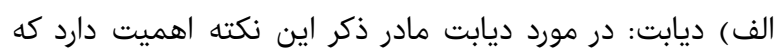

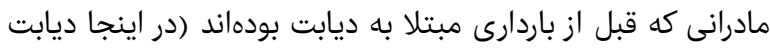

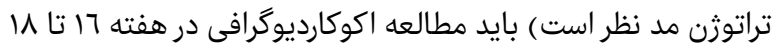

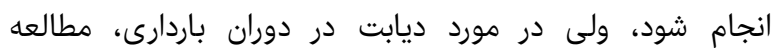

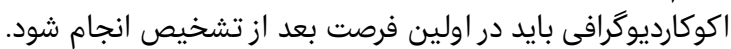

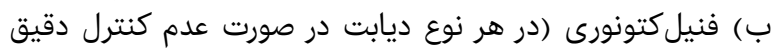

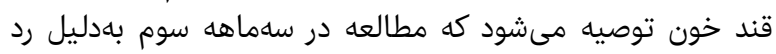

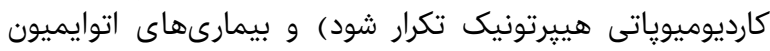

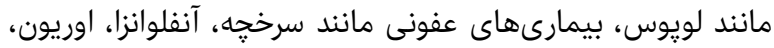

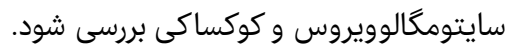

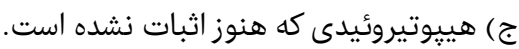

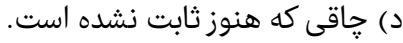

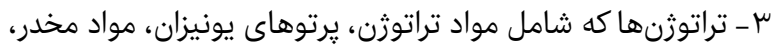

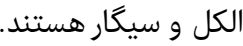

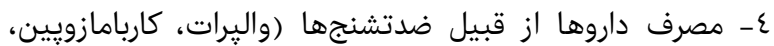

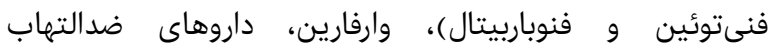

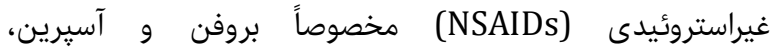

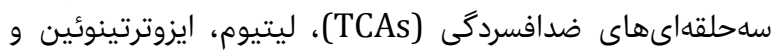

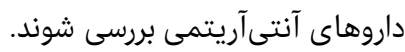
IVF -0 7 - وجود اختلالات كروموزومى رجى يا ناهنجارىهاى خارى خارج قلبى در

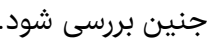
- V

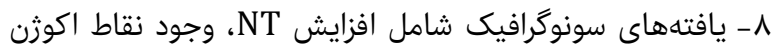

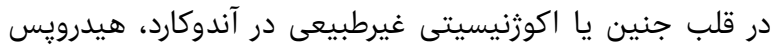

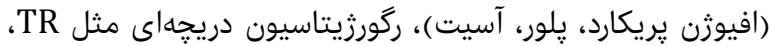

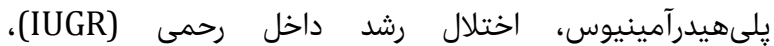

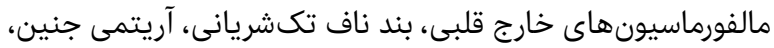


ظاهرى طولانى خواهد داشت؛ هر حند اين كار قابل انجام باشد،

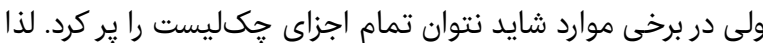

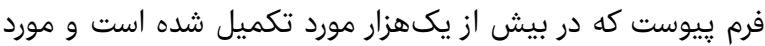

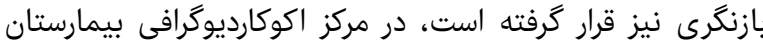
صارم كاربرد دارد و ييشنهاد مى شود.

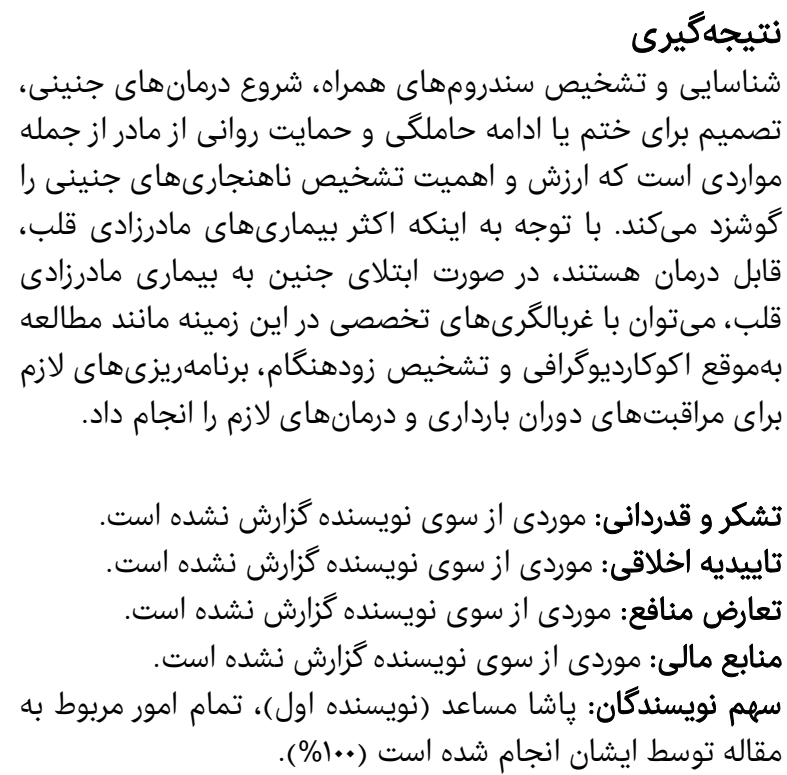

1- Drose JA. Fetal echocardiography. Amsterdam: Elsevier Health Sciences; 2013.

2- Carvalho J, Allan L, Chaoui R, Copel J, DeVore G, Hecher $\mathrm{K}$, et al. ISUOG Practice guidelines (updated): sonographic screening examination of the fetal heart. Ultrasound Obstet Gynecol. 2013;41(3):348-59.

3- Satomi G. Guidelines for fetal echocardiography. Pediatr Int. 2015;57(1):1-21.

4- Creasy RK, Resnik R, Iams JD. Maternal-fetal medicine: Principles and practice. Houston: Gulf Professional Publishing; 2004.

5- Rychik J, Ayres N, Cuneo B, Gotteiner N, Hornberger L, Spevak PJ, et al. American society of echocardiography guidelines and standards for performance of the fetal echocardiogram. J Am Soc Echocardiogr. 2004;17(7):803-10.

6- Fetal Echocardiography Task Force; American Institute of Ultrasound in Medicine Clinical Standards Committee; American College of Obstetricians and Gynecologists; Society for Maternal-Fetal Medicine. Practice guideline for the performance of fetal echocardiography. J Ultrasound Med. 2011;30(1):127-36.
11- Aortic Arch (Race Tract) V

12- Ductal Arch V

13- Ventricular Septum Subcoital V

14- Doppler Exam of AV Valves

15- Doppler Exam of Semi lunar Valves (Out flow pattern)

16- $M$ mode of Ventricular Wall

17- Doppler Exam of Foramen Ovale

$$
\text { با نماها و تصاوير فوق، بايد بتوان موارد زير را بهدست آورد: }
$$

1- Fetal Number

2- Fetal Position

3- Visceral situs

4- Cardiothorasic ratio

5- Atrioventricular and Ventricularterial Connection

6- Pulmonary Veins (Number and Return)

7- IVC and SVC

8- Foramen Ovale (FO)

9- Ductus Venosis (DV)

10- Umblicle Arteries and Vein

11- Chambers Position and situs and size

12- AV Valves (Size and Flew)

13 Semi Lunar Valves (Size and Flew)

14- Pulmonary Artery (Size and Branching)

15- Aorta and Aortic Arch

16- Ductal Arch

17- Ductus Flow Pattern

18- Interventricular Septum (Size and Integrity)

19- Fetal Heart Rate (FHR)

20- Atrial and Ventricular Contraction and Conduction

21- Fractional Shortening (FS)

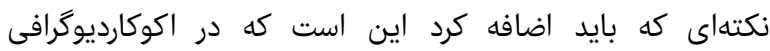

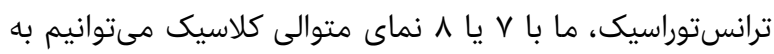

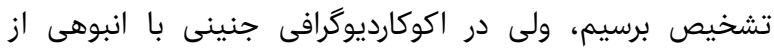

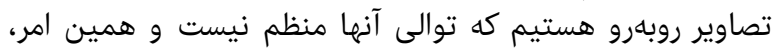

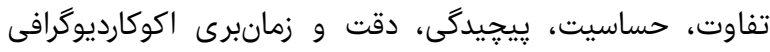

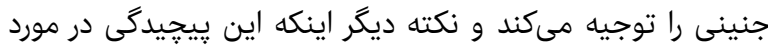

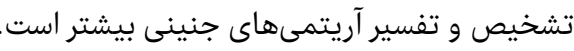

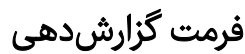

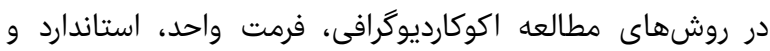

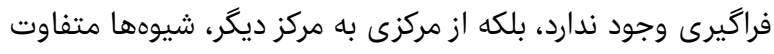

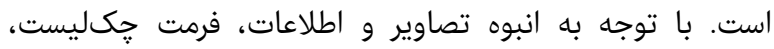

